\title{
Kinteract: A Multi-sensor Physical Rehabilitation Solution based on Interactive Games
}

\author{
Nuno Matos \\ nuno.matos@fraunhofer.pt
}

\author{
António Santos \\ antonio.santos@fraunhofer.pt \\ Fraunhofer Portugal AICOS \\ Rua Alfredo Allen 455 \\ 4200-135 Porto, Portugal
}

\author{
Ana Vasconcelos \\ ana.vasconcelos@fraunhofer.pt
}

\begin{abstract}
All over the world there are millions of people who are living with long-term motor impairments caused by a stroke or any other kind of corticospinal tract injuries. The physical rehabilitation of these patients is usually slow and demotivating. In this paper we introduce Kinteract, a novel solution that applies the paradigm of using motion-based games in the rehabilitation process, with the added value of providing a motion sensor server that supports a growing array of motion sensors (currently Microsoft Kinect, Leap Motion and Orbotix Sphero) and merge their data into a single protocol that can be used for any purpose. The use of different sensors, even at the same time, allows the rehabilitation of specific parts of the body. This data can be stored in a server for physicians to analyse and can clearly reveal the evolution of the patient in the rehabilitation process.
\end{abstract}

\section{Categories and Subject Descriptors}

H.1.2 [User/Machine Systems]: Human Factors; J.3 [Life and Medical Sciences]: Health

\section{General Terms}

Design, Experimentation, Human Factors, Measurement

\section{Keywords}

Physical Rehabilitation, Stroke, Virtual Reality, Natural User Interfaces, Kinect, Leap Motion, Sphero

\section{INTRODUCTION}

According to the World Health Organization [1], 15 million people suffer a stroke worldwide each year. Of these, 5 million die and another 5 million are permanently disabled. About $80 \%$ of people who survive a stroke experience motor impairments. One such impairment is hemiparesis: a partial paralysis of one side of the body [7]. Hemiparesis usually causes chronic disability in the upper extremities (arms)

Permission to make digital or hard copies of all or part of this work for personal or classroom use is granted without fee provided that copies are not made or distributed for profit or commercial advantage and that copies bear this notice and the full citation on the first page. To copy otherwise, to republish, to post on servers or to redistribute to lists, requires prior specific permission and/or a fee. REHAB 2014, May 20-23, Oldenburg, Germany Copyright (c) 2014 ICST 978-1-63190-011-2 DOI 10.4108/icst.pervasivehealth.2014.255325 more than the lower extremities (legs). People with hemiparesis experience limitations in fine motor control, strength, and range of motion [2].

Repetitive exercises can provide the brain with sufficient stimuli to remodel itself and provide better motor control [6]. However, only $31 \%$ of stroke patients perform these exercises as recommended [12], which can lead to an incomplete recovery [8]. Therefore, finding motivating and effective ways to encourage people with hemiparesis to perform therapeutic exercises is crucial in helping them achieve a more complete recovery.

Video games with motion-based input devices may provide a motivating way to help people with hemiparesis complete therapeutic exercises and regain lost motor control [3][4]. In addition, game systems should monitor and measure users' motion abilities and detect when improvements occur in both supervised and unsupervised settings.

Due to the ever growing set of affordable and commercially available motion sensing devices, it is now being perceived by therapists that they may be used for physical rehabilitation purposes. With this work, the authors introduce a novel physical rehabilitation solution comprised of several games that can use different motion sensors (even simultaneously), being their data provided by a sensor server and using a common protocol.

\section{STATE OF THE ART}

The use of IT in physical rehabilitation programs is not new and is making huge improvements in terms of effective results.

VirtualRehab [13] is a VirtualWare product developed for PC that uses Microsoft Kinect to track and capture the movements of the patients and to allow them to play the provided games. These games are particularly focused on the balance, coordination and posture of the patients and use customized rehabilitation programs to help treat these physical symptoms. Thanks to a cloud based platform, there is also the possibility of registering the results obtained each time a patient plays one of the games so the therapist can be always supervising his patients' evolution whether they are having their sessions at a clinic center or at home. REMOVIEM [9] is a very recent project that is being used in the rehabilitation of patients from the Association of Multiple Sclerosis of Castellon and also promotes body move- 
ments, tracked by the Kinect sensor, through three motor rehabilitation games that try to improve the gait, the balance and weight transfer of the patient. Kitsunezaki et al [5] implemented a solution that uses the Kinect sensor in a quite different way. This solution is based on an exercise that requires two sensors and two $\mathrm{PCs}$, connected via an Ethernet hub, and where the user must cross the radius of action of both motion sensor devices by starting in front of one sensor and walking about ten meters until he reaches the other one. This exercise will allow the measurement of the step frequency, step length and velocity of the patient when doing the route, three essential gait parameters [10], with the help of the system's PCs that record the time spent on the exercise. Another different usage for this device was proposed by Saini et al [11] and consists in a home-based platform that gathers an online biofeedback component to the movement tracking and exercises, which allows the system to store the patient's movements and knowledge result and display them to both patient and therapist. This type of solution is convenient for the patient because he can have his sessions at home without the need of his therapist.

\section{ARCHITECTURE}

When this project started to take shape, it was decided that one of the requirements was to have the ability to provide motion sensor data to any application that implements a Natural User Interface. Therefore, it was decided to split the solution in two main components: a motion sensor server, providing sensor data using a single protocol and through a common communication mechanism, and the client, in this case the application that runs the rehabilitation games and which processes the incoming sensor data. As such, this model allows the server to be expanded without affecting the client applications or be reused in different contexts.

\subsection{Server}

Instead of having to dig deep in the SDK of a specific motion sensor and limit the support to just that sensor, application developers can instead use this server and support the used protocol, no matter which sensor is being used. And if at the beginning the server only supported the Microsoft Kinect, now it currently supports other completely different sensors like the Leap Motion and Orbotix Sphero. Furthermore, the applications can use more than one sensor simultaneously using the same protocol (Fig. 1).

\subsubsection{Supported sensors}

As mentioned previously, the development of the server started by providing support to the well-known Microsoft Kinect sensor. This sensor, apart from the video stream provided by the included RGB camera, provides a depth map that is obtained through the use of a IR light emitter and receiver. This allows software frameworks to be able to detect bodies in the field-of-view and track their skeleton through the detection of 20 joints. Through the use of a common SDK (OpenNI/NiTE), the support was expanded to other similar sensors like the Asus Xtion Pro.

Later, with the release of the Leap Motion, it was inevitable to add support to this sensor due to its characteristics. Leap Motion allows a real-time tracking of the hands and pointables (it may be fingers or other tools like pens) (Fig. 2). The

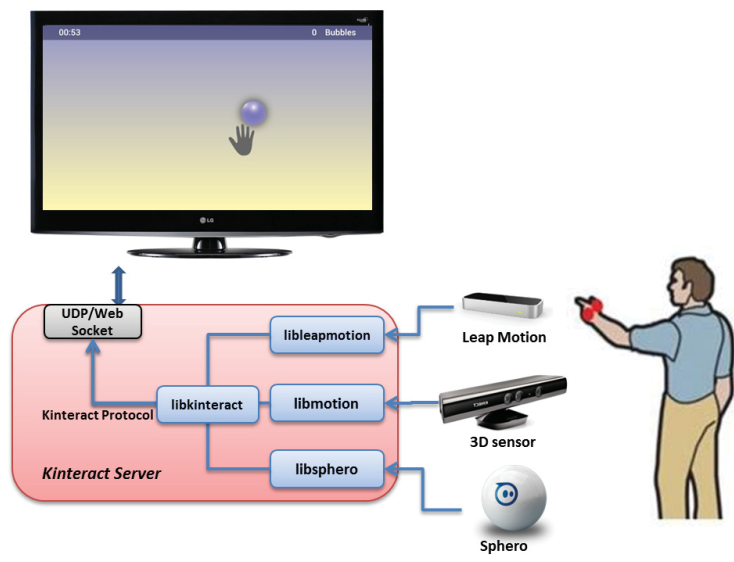

Figure 1: Kinteract Server Architecture

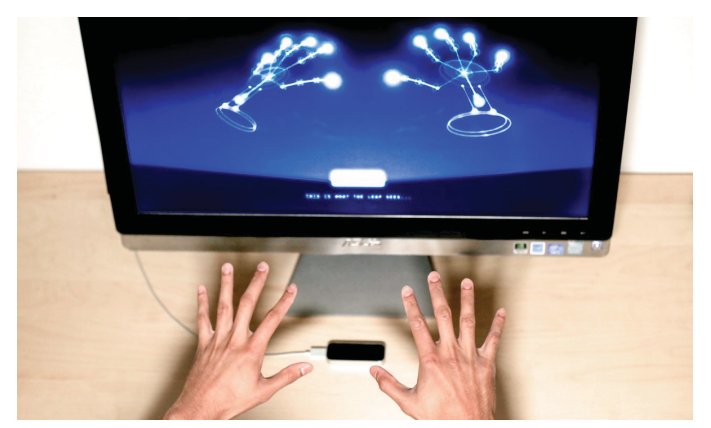

Figure 2: Leap Motion

sensor provides precise values regarding the position and orientation of the hands and pointables, allowing not only the tracking of these objects but also the detection of gestures. The main drawback is the limited field of view, which only allows the detection of hands up to $60 \mathrm{~cm}$ from the sensor. However, it brings clear advantages for the rehabilitation of the hand.

Up to this moment, the Kinteract server was added with the support to another sensor, the Orbotix Sphero. This device is mostly well known as being a "robotic ball", with nearly the size of a tennis ball and featuring Bluetooth communication and being able to be controlled by a mobile device, but it can also be used as a controller due to the powerful IMU it has integrated. Therefore, it can also be used as a motion sensor and may be applied in monitoring some hand movements like rotation.

\subsubsection{Architecture}

The Kinteract server was designed to fit in every context, being provided as shared library that can be easily integrated into an application under development or providing a serverclient model, opening an UDP/Web socket to receive connections from clients and providing a stream of motion sensor data. Although this is an unlikely scenario, the server supports several clients at the same time. Developed in $\mathrm{C}++$, it currently runs on Windows and Linux and the support to the different sensors is provided through individual shared libraries/DLLs, which are dynamically loaded on demand. 


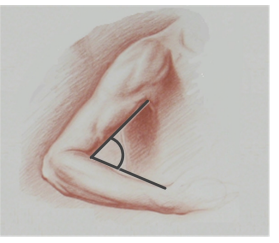

(a) Angle between arm and forearm

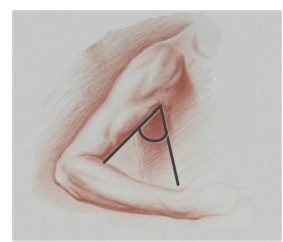

(b) Angle between arm and torso
Figure 3: Measured angles in Chasing Bubbles and Space Lights

The server is highly configurable and may easily be adapted to the clients needs. Apart from normal hand tracking, the server is able to detect common gestures with both $3 \mathrm{D}$ motion sensor and Leap Motion. The server is also able to perform open/closed hand detection with 3D motion sensor by applying Computer Vision algorithms implemented using OpenCV, although the accuracy is still not optimal.

\subsection{Client}

The client component of this solution is an Android application running on Google TV providing a platform for physical rehabilitation games. The application features a background service that is in charge of processing the sensor data sent using the Kinteract protocol. The user can either use Google TV's remote control or one of the available sensors to navigate the menus and may play the games as a guest or as a registered player if an external Personal Health Record is being used to manage patient profiles and store measurements. When playing as a guest, there will be no player profile and the results will not be saved. Several difficulty levels are available, each one with different combinations of available time and goal, which allow keeping up with the evolution of the patient and keep challenging him.

\subsubsection{Chasing Bubbles}

This game is played with the 3D motion sensor (Xtion Pro / Kinect) and its goal is to reach a specific number of bubbles (one at a time) that keep appearing on the TV screen in the shortest amount of time. Depending on the difficulty level, the bubbles will appear randomly in a specific distance interval from the center of the screen. The idea is to promote wide arm movements and monitor the angle between the torso and the arm and the one between the arm and the forearm (Fig. 3).

\subsubsection{Space Lights}

This game is similar to the previous one and also aims at monitoring the aforementioned angles. The main difference is that now the game goal is achieved in two steps, since the user must first pick a light, standing his hand over it for roughly 1.5 seconds, and drag it into a black hole on a different position on the screen (Fig. 4). This leads to a bigger physical effort from the user.

\subsubsection{Dragging Apples}

Unlike the first two, this third game is played with the Leap Motion sensor and mainly targets the rehabilitation of the hand, trying to increase its opening range. With the 3D motion sensor is not possible to measure this but the Leap

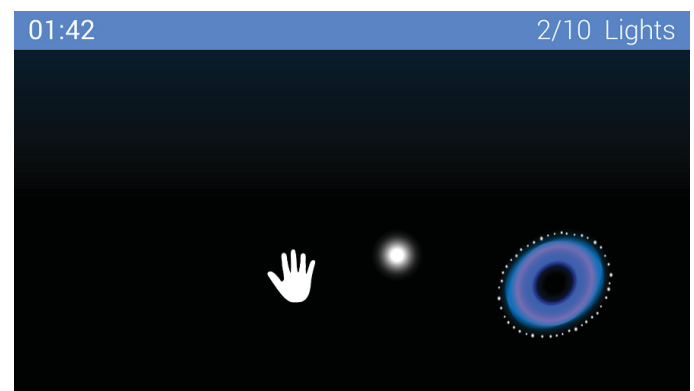

Figure 4: Space Lights game

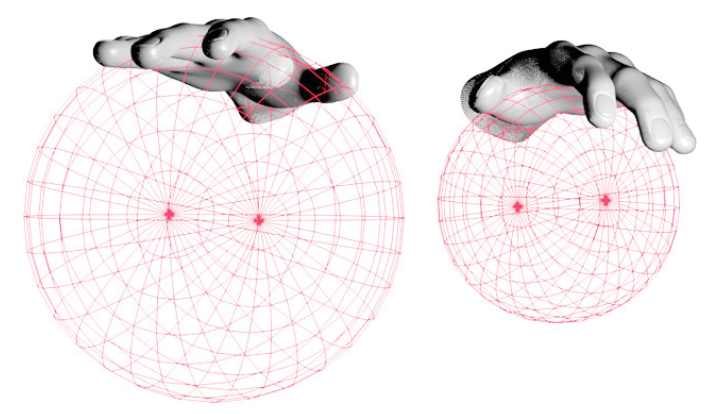

Figure 5: Leap Motion virtual sphere (source: Leap Motion Developer Documentation)

Motion sensor does a great job here. The goal of the game is to pick an apple standing on a specific position of the screen by opening the hand by a certain extent, drag the apple to a basket (still with the hand opened) and drop it by closing the hand. Leap Motion can provide the radius of a virtual sphere that fits the curvature of the hand (Fig. 5 ) and this value is used as the main metrics to assess the patient's evolution. The difficulty level is also proportional to the minimum sphere radius needed to open the hand.

\section{PERFORMANCE AND RESULTS}

Two sets of tests were made with five healthy users (HU) and five patients (IU) with upper limb hemiparesis at Centro de Reabilitação Profissional de Gaia (CRPG) (Gaia's Professional Rehabilitation Center). Only the first two games mentioned in 3.2 were tested, since the third one using Leap Motion was not ready at the time. From these five participants, only one had some experience using motion sensors applied to interactive games.

Both sets had three different sessions, each one with different difficulty levels. The first set consisted in playing the Chasing Bubbles game. This game was easily understood by all participants considering there were no mistakes and not a single assistance was given in any of the sessions. The second set (Space Lights) was a bit more difficult to be understood and played due to its characteristics. $80 \%$ of the users needed assistance with this game.

Despite those difficulties, the efficiency results for each game were good as well as the overall satisfaction. All ten users were able to achieve the goals for each game. The game results are detailed in Table 1 and Table 2 and clearly reveal 
the increasing difficulty between the different test sets. It can be spotted on Table 2 the considerably longer time taken to pick the object when compared to the time taken to drag it, due to the extra effort needed to stand the arm for 1.5 seconds.

\begin{tabular}{|c|c|c|c|c|c|c|}
\hline & \multicolumn{6}{|c|}{ Chasing Bubbles } \\
\hline & \multicolumn{2}{|c|}{$1^{\text {st }}$ Test } & \multicolumn{2}{|c|}{$2^{\text {nd }}$ Test } & \multicolumn{2}{|c|}{$3^{\text {rd }}$ Test } \\
\hline & ANB & ATS & ANB & ATS & ANB & ATS \\
\hline IU & $\overline{42}$ & 1.34 & 32 & 1.82 & 13 & 2.44 \\
\hline HU & 61 & 0.80 & 61 & 0.96 & 25 & 1.02 \\
\hline
\end{tabular}

Table 1: Efficiency on Chasing Bubbles (ANB - Average Number of caught Bubbles ; ATS - Average Time Spent (secs)

\begin{tabular}{|c|c|c|c|c|c|c|c|c|c|}
\cline { 2 - 11 } \multicolumn{1}{c|}{} & \multicolumn{9}{c|}{ Space Lights } \\
\cline { 2 - 11 } \multicolumn{1}{c|}{} & \multicolumn{3}{c|}{$1^{\text {st }}$ Test } & \multicolumn{3}{c|}{$2^{\text {nd }}$ Test } & \multicolumn{3}{c|}{$3^{\text {rd }}$ Test } \\
\cline { 2 - 12 } & ANL & ATSP & ATSD & ANL & ATSP & ATSD & ANL & ATSP & ATSD \\
\hline IU & 8 & 5.01 & 2.51 & 7 & 4.40 & 2.87 & 2 & 7.81 & 2.62 \\
\hline HU & 15 & 2.94 & 1.16 & 15 & 4.05 & 1.00 & 6 & 3.25 & 1.09 \\
\hline
\end{tabular}

Table 2: Efficiency on Space Lights (ANL - Average Number of dragged Lights; ATSP - Average Time Spent Picking (secs); ATSD - Average Time Spent Dragging (secs)

Regarding user satisfaction, each patient was asked to fill in a SUS (System Usability Scale) questionnaire at the end of the performed tests and the results are shown on Table 3.

\begin{tabular}{|l|r|}
\hline Participants & Avg. score (max. 100) \\
\hline IU & 87.5 \\
HU & 91.5 \\
\hline
\end{tabular}

Table 3: Usability questionnaire results

\section{CONCLUSION AND FUTURE WORK}

The presented solution, as a whole, had a good reaction and feedback from the patients who tested it and, although there are still no quantitative data regarding the impact of the use of the solution on the rehabilitation process, there is now a clear road ahead. One of the issues found is the login gesture. Due to their condition, patients may have difficulties in making the login gesture (which may be a wave, a click/push or a hand raise gesture), which is needed to start the tracking with the $3 \mathrm{D}$ motion sensor.

The choice for an Android application running on Google $\mathrm{TV}$ also proved not to be quite practical in terms of deployment, since there is the need to have a second device running the server (the server does not run on Android devices) with a proper network setup. Therefore, one of foreseen developments is to implement the client application using the Unity framework, which will allow building releases for different platforms (including Android, which runs on Google TV), and will allow running the client application and the server in the same device (Windows or Linux).

To assess the feasibility and effectiveness of the solution in the rehabilitation process, long term tests are planned. There are also plans to widen the array of supported sensors, namely with the inclusion of the Myo sensor, as well as improving posture and gesture recognition algorithms. New games focusing on the rehabilitation of the lower limb will also broaden the spectrum of the solution.

\section{ACKNOWLEDGEMENTS}

The authors would like to thank CRPG, namely Dra. Cristina Crisóstomo and Dra. Alexandra Couto, for their support, and acknowledge the financial support from NSFR/ERDF/EU.

\section{REFERENCES}

[1] The world health report 2002 - Reducing Risks, Promoting Healthy Life. World Health Organization, 2002.

[2] G. Alankus, R. Proffitt, C. Kelleher, and J. R. Engsberg. Stroke therapy through motion-based games: A case study. TACCESS, 4(1):3, 2011.

[3] P. Bach-y Rita, S. Wood, R. Leder, O. Paredes, D. Bahr, E. Bach-y Rita, and N. Murillo. Computer-assisted motivating rehabilitation (camr) for institutional, home, and educational late stroke programs. Topics in Stroke Rehabilitation, 8(4):1-10, 2002.

[4] J. Deutsch, J. Latonio, G. Burdea, and R. Boian. Post-stroke rehabilitation with the rutgers ankle system: A case study. Presence, 10(4):416-430, August 2001.

[5] N. Kitsunezaki, E. Adachi, T. Masuda, and J.-i. Mizusawa. Kinect applications for the physical rehabilitation. In Medical Measurements and Applications Proceedings (MeMeA), pages 294-299. IEEE, 2013.

[6] J. Kleim, T. Jones, and T. Schallert. Motor enrichment and the induction of plasticity before or after brain injury. Neurochemical Research, 28(11):1757-1769, November 2003.

[7] P. Langhorne, F. Coupar, and A. Pollock. Motor recovery after stroke: a systematic review. Lancet neurology, 8(8):741-754, 2009.

[8] M. Lotze and L. G. Cohen. Volition and imagery in neurorehabilitation. Cognitive and behavioral neurology, 19(3):135-140, 2006.

[9] J. Lozano-Quilis, H. Gil-Gómez, J. Gil-Gómez, S. Albiol-Pérez, G. Palacios, H. M. Fardoum, and A. S. Mashat. Virtual reality system for multiple sclerosis rehabilitation using kinect. In Pervasive Computing Technologies for Healthcare (PervasiveHealth), pages 366-369. IEEE, 2013.

[10] T. Öberg, A. Karsznia, and K. Öberg. Basic gait parameters: reference data for normal subjects, 10-79 years of age. Journal of rehabilitation research and development, 30:210-210, 1993.

[11] S. Saini, D. R. A. Rambli, S. Sulaiman, M. N. Zakaria, and S. R. M. Shukri. A low-cost game framework for a home-based stroke rehabilitation system. In Computer E Information Science (ICCIS), volume 1, pages 55-60. IEEE, 2012.

[12] M. Shaughnessy, B. Resnick, and R. Macko. Testing a model of post-stroke exercise behavior. Rehabilitation Nursing, 31(1), January/February 2006.

[13] VirtualWare Group. Virtualrehab. http://virtualrehab.info/, 022014. 